\title{
Prevalence and Implications of Bone Marrow Involvement in Patients with Gastric Mucosa-Associated Lymphoid Tissue Lymphoma
}

\author{
Sang II Choi ${ }^{1}$, Myeong-Cherl Kook ${ }^{1}$, Sanghyun Hwang ${ }^{2}$, Young-II Kim ${ }^{1}$, Jong Yeul Lee ${ }^{1}$, Chan Gyoo Kim ${ }^{1}$, II Ju Choi ${ }^{1}$, \\ Hyewon Lee ${ }^{3}$, Hyeon Seok Eom ${ }^{3}$, and Soo-Jeong Cho ${ }^{1}$ \\ ${ }^{1}$ Center for Gastric Cancer, National Cancer Center, Goyang, ${ }^{2}$ Department of Laboratory Medicine, Asan Medical Center, Seoul, and ${ }^{3}$ Center for \\ Hematologic Malignancy, National Cancer Center, Goyang, Korea
}

Background/Aims: Mucosa-associated lymphoid tissue (MALT) lymphoma of the stomach is an uncommon disease. Bone marrow involvement is reported even in patients with only a mucosal lesion. We evaluated the prevalence and risk factors of marrow involvement and its implications for diagnosis and treatment. Methods: In total, 132 patients who were diagnosed with gastric MALT lymphoma at the National Cancer Center in Korea between January 2001 and December 2016 were enrolled in the study. The patient data were collected and analyzed retrospectively. Results: Of the 132 patients, 47 (35.6\%) were male, with a median age of 52 years (range, 17 to 81 years). The median follow-up duration was 48.8 months (range, 0.5 to 169.9 months). Helicobacter pylori infection was detected in 82 patients (62.1\%). Most patients $(80.3 \%)$ had stage IE1 according to the modified Ann Arbor staging system. Ninety-two patients underwent bone marrow evaluation, and four patients (4.3\%) had marrow involvement. Of these patients, one presented with abdominal lymph node involvement, while the other three had stage IE1 disease if marrow involvement was disregarded. All three patients had no significant symptoms and were monitored after local treatment without evidence of disease aggravation. Conclusions: Bone marrow involvement was found in $4.3 \%$ of the patients with gastric MALT lymphoma. Bone marrow examination may be deferred because marrow involvement does not change the treatment options or outcome in gastric MALT Iymphoma confined to the stomach wall. (Gut Liver 2018;12:278-287)

Key Words: Lymphoma, B-cell, marginal zone; Bone marrow involvement; Helicobacter pylori; Prognosis

\section{INTRODUCTION}

Mucosa-associated lymphoid tissue (MALT) lymphoma is currently classified as an extranodal marginal zone lymphoma according to the Revised European American Lymphoma classification system ${ }^{1}$ and the classification proposed by the World Health Organization (WHO). ${ }^{2}$ Among the three subtypes of marginal zone lymphoma according to the international lymphoma study group classification of non-Hodgkin's lymphoma, ${ }^{3}$ which are extranodal, nodal, and splenic marginal zone lymphoma, extranodal marginal zone lymphoma of MALT type is known to be the most common subtype, accounting for 50\% to $70 \%$ of total cases.

MALT lymphoma is induced by chronic inflammatory process, resulting in accumulation of autoreactive lymphoid tissue around the germinal centers in Peyer's patches, thus named the marginal zone. This process may be associated with chronic infectious condition such as Helicobacter pylori infection in stom$\mathrm{ach}^{4}$ and autoimmune disease in thyroid or salivary gland., These autoreactive cells transform into MALT lymphoma sometimes with the acquisition and accumulation of genetic abnormalities such as $t 11 ; 18$ and $t 1 ; 14 .^{7}$ MALT lymphoma may arise from gastrointestinal track as well as from non-gastrointestinal sites such as conjunctivae, thyroid, salivary gland, orbit, lung, breast, kidney, skin, liver, and prostate. However, stomach is known to be the most common site associated with $H$. pylori infection. ${ }^{7,8}$

MALT lymphoma is considered to be an indolent lymphoma with an excellent prognosis due to good clinical responses to treatment and favorable disease-free and overall survival. This is probably partly because MALT lymphoma tends to stay localized for a prolonged period of time without dissemination. ${ }^{9}$

Correspondence to: Soo-Jeong Cho

Center for Gastric Cancer, National Cancer Center, 323 Ilsan-ro, Ilsandong-gu, Goyang 10408, Korea

Tel: +82-31-920-1604, Fax: +82-31-920-1289, E-mail: crystal522@ncc.re.kr

Received on May 15, 2017. Revised on September 21, 2017. Accepted on September 30, 2017. Published online February 8, 2018 pISSN 1976-2283 eISSN 2005-1212 https://doi.org/10.5009/gnl17217

@ This is an Open Access article distributed under the terms of the Creative Commons Attribution Non-Commercial License (http://creativecommons.org/licenses/by-nc/4.0) which permits unrestricted non-commercial use, distribution, and reproduction in any medium, provided the original work is properly cited. 
However, its spread to regional lymph nodes and to multiple sites occurs in some cases. Furthermore, bone marrow involvement is reported in $7 \%$ to $20 \%$ of the total cases of MALT lymphoma. ${ }^{10,11}$

In gastric MALT lymphoma, bone marrow involvement is reported in some cases, even in patients with only mucosal lesions without lymph node involvement, ${ }^{12-14}$ but their incidence has not been clearly presented yet. There are several clinical guidelines for the diagnosis and treatment of MALT lymphoma, which are similar but different especially in regard to the study of bone marrow involvement. ${ }^{15-17}$ Due to strong association between $H$. pylori infection and MALT lymphoma, the incidence and the clinical characteristics may show variations in different parts of world according to its endemicity, potentially limiting the value of the clinical guidelines. Thus, we tried to evaluate the incidence of bone marrow involvement in patients with gastric MALT lymphoma and its treatment options especially for early stage disease with bone marrow involvement through a retrospective analysis of the experience in a single center in South Korea.

\section{MATERIALS AND METHODS}

This study has been approved by the Institutional Review Board of the National Cancer Center, Goyang, South Korea (NCC2017-0105). Patient consent was waived according to the approval of Review Board since the right and the interest of the patients would not be violated.

\section{Patients}

Between January 2001 and December 2016, 144 patients were diagnosed with gastric MALT lymphoma at the National Cancer Center, Korea and were followed at two centers: center for gastric cancer and center for hematologic malignancy. Patients who had definite diagnosis upon pathologic evaluation through endoscopic biopsy in our institute were included in this study. Patients who were diagnosed but not treated or followed at current institute were excluded. In addition, those with other invasive malignancies or malignant transformation of MALT lymphoma at the time of initial diagnosis were excluded from the study.

Information, including baseline characteristics, disease history and status, treatment and its outcome, was retrospectively collected using electronic medical records. Patients were followed until January 2017

\section{Diagnosis and staging procedure}

Complete physical exam was done at the time of initial presentation. Grade 4 to 5 according to histologic scoring system suggested by Wotherspoon et al. ${ }^{18}$ was considered to be compatible with diagnosis of MALT lymphoma. Polymerase chain reaction (PCR) for immunoglobulin heavy chain ( $\mathrm{IgH}$ ) was used in adjunction to pathologic evaluation to support diagnosis. If a histology showed dense infiltrate of marginal zone cells in lamina propria with prominent lymphoepithelial lesions (Wotherspoon score 5), the cases were diagnosed as MALT lymphoma. If a histology was ambiguous for diagnosis, we performed PCR examination. Wotherspoon scores 3 or 4 combined with IgH monoclonality from PCR was also considered to be MALT lymphoma. Additionally, when the histologic evidences (dense infiltrates, multiple lymphoepithelial lesions) and endoscopic lesions were highly suggestive, the cases were diagnosed as MALT lymphoma regardless of the PCR results.

Upon confirmation of MALT lymphoma on endoscopic biopsy of the stomach, further evaluation for staging of the disease was done: computed tomography (CT) of chest, abdomen and pelvis and laboratory tests including blood count, lactase dehydrogenase (LDH), and $\beta 2$-microglobulin. Endoscopic ultrasound (EUS) was carried out in selected patients to evaluate the depth of invasion of MALT lymphoma. Bone marrow aspiration and biopsy and positron emission tomography (PET) were performed at the discretion of each attending physician to confirm marrow involvement and involvement of other sites. PCR for IgH was also performed on bone marrow aspiration samples if available.

Bone marrow evaluation was done according to the usual procedure in a prone position. ${ }^{19}$ Bone marrow involvement was confirmed when definite evidence of nodular, nodular and interstitial or paratrabecular lymphoid infiltration was found on bone marrow biopsy. ${ }^{20}$

Staging of the disease followed modified Ann Arbor staging system for extranodal lymphoma. ${ }^{17,21}$ Stage IIE1 referred to disease with regional lymph node involvement, while IIE2 referred to disease with distant abdominal lymph node involvement. Paris staging system was used in combination with Ann Arbor system to better delineate the depth of disease involvement. ${ }^{22}$ Because bone marrow involvement is sufficient to stage a patient to stage IV MALT lymphoma according to current staging system, in order to compare the baseline characteristics of patients with and without bone marrow involvement, we additionally staged the patients disregarding bone marrow involvement.

Endoscopic finding was primarily based on the endoscopy report at the time of initial diagnostic work up; however, previous endoscopy images were also reviewed if sufficient information was not available in the report. When more than two discrete lesions with intact normal mucosa in between were confirmed as MALT lymphoma through endoscopic biopsy at each site, the patient was considered to have multiple lesions. H. pylori infection status was evaluated using rapid urease test, pathologic evaluation with Wright-Giemsa staining of sample which was acquired from endoscopic biopsy at greater curvature side of the body of the stomach, serology test, or urea breath test. Infection with $H$. pylori was confirmed if any of the above tests turned out positive.

Patients were considered to have lymph node involvement if 
lymph node enlargement of more than $1.0 \mathrm{~cm}$ in short axis was noted on the CT $\operatorname{scan}^{23}$ or if the PET scan showed significant uptake.

\section{Treatment}

Patients were treated according to $H$. pylori infection status, disease stage and disease location. Combination of amoxicillin (1,000 mg twice a day) and clarithromycin (500 mg twice a day) with high-dose proton pump inhibitor (omeprazole $20 \mathrm{mg}$, lansoprazole $30 \mathrm{mg}$, or pantoprazole $40 \mathrm{mg}$ twice a day) for 7 to 14 days or combination of metronidazole $(500 \mathrm{mg}$ three times a day), tetracycline (500 mg four times a day) and bismuth (600 mg twice a day) with high-dose proton pump inhibitor for 10 days was used to treat patients with $H$. pylori infection. For patients without $H$. pylori infection, radiation therapy was used if the patient had disease confined to gastric wall, and systemic chemotherapy was used if the patient presented with lymph node involvement.

\section{Follow-up evaluation and second-line treatment}

Follow-up tests to see the $H$. pylori infection status were also carried out to evaluate the response to the $H$. pylori eradication treatment. Bone marrow aspiration and biopsy was performed at the discretion of each attending physician if the patient had bone marrow involvement at initial presentation.

After initial treatment, follow-up upper endoscopy with biopsy was performed every 3 to 6 months and pathologic evaluation for biopsy sample was done using the Groupe d'Etude des Lymphomes de I'Adulte (GELA) histological grading system. ${ }^{24}$ Complete remission (CR) was defined when there was no evidence of endoscopic and pathologic evidence of disease in two post-treatment evaluations that was done at least 4 weeks apart.

Second-line treatment was administered according to followup H. pylori infection status, disease status after initial treatment, presence of systemic symptoms and the performance status of the patient.

\section{Statistical analysis}

Proportion of patients with bone marrow involvement was calculated from the patients who underwent bone marrow evaluation at the time of initial diagnostic work up. Baseline characteristics were compared between the patients who had and those that did not have bone marrow involvement. Student t-test and Fisher exact test were used to compare age, LDH level, and $\beta 2$-microglobulin level at the time of initial diagnosis. Proportion of patients with $H$. pylori infection, multiple lesions and lymph node involvement were also compared. Pearson chisquare test was used to compare $\mathrm{T}$ stage between the groups. Significant difference was defined as p-value less than 0.05. All statistical analyses were performed by using Stata/SE software version 14.1 (StataCorp LP, College Station, TX, USA).

\section{RESULTS}

\section{Baseline characteristics}

Among the 144 patients who were screened for this study, 12 patients were excluded due to various reasons: five patients were diagnosed but not treated or followed at current institute, six had other invasive malignancies, and one exhibited malignant transformation on the endoscopic biopsy at the time of initial diagnosis. As a result, 132 patients were included in this study (Fig. 1).

Baseline characteristics of all patients in current study are shown in Table 1. Median age was 52 years old, ranging from 17 to 81 , and the majority of patients were below 65 (116 patients, 88.8\%). Female predominance was noted with femaleto-male ratio of 1.81. Patients were followed for median of approximately 48.8 months (range, 0.5 to 169.9 months).

Upon initial endoscopic evaluation, multiple lesions were confirmed in 63 patients (47.7\%), while other patients had solitary lesion associated with MALT lymphoma. MALT lymphoma was most frequently found at body of stomach followed by

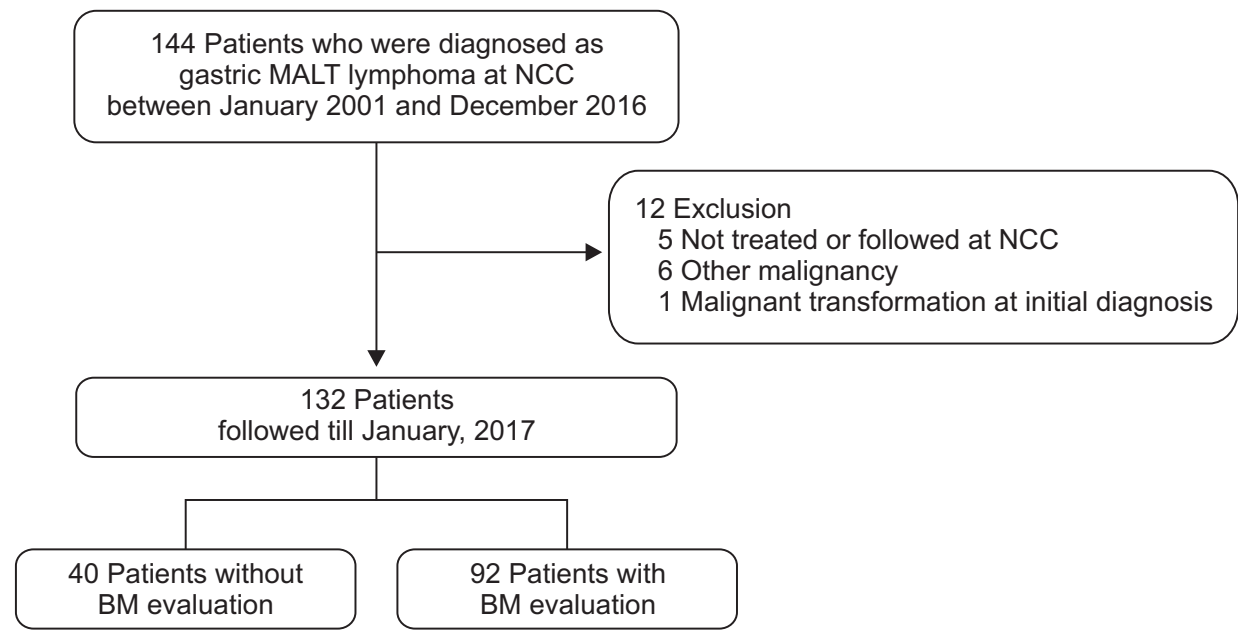

Fig. 1. Study design.

MALT, mucosa-associated lymphoid tissue; NCC, National Cancer Center; $\mathrm{BM}$, bone marrow. 
Table 1. Baseline Characteristics ( $\mathrm{n}=132)$

\begin{tabular}{|c|c|}
\hline Variable & Value \\
\hline Age, yr & $52(17-81)$ \\
\hline Sex, male/female & $47(35.6) / 85(64.4)$ \\
\hline Follow-up duration, mo & $48.8(0.5-169.9)$ \\
\hline Multiplicity & $63(47.7)$ \\
\hline \multicolumn{2}{|l|}{ Location of lesion } \\
\hline Antrum & $23(17.4)$ \\
\hline Angle & $9(6.8)$ \\
\hline Body & $55(41.7)$ \\
\hline Fundus & $8(6.1)$ \\
\hline Multiple sites & $37(28.0)$ \\
\hline \multicolumn{2}{|l|}{ Endoscopic finding } \\
\hline Mucosal change & $52(39.4)$ \\
\hline Ulcer & $30(22.7)$ \\
\hline Erosion & $22(16.7)$ \\
\hline Nodular elevation & $22(16.7)$ \\
\hline Mass & $4(3.0)$ \\
\hline Polyp & $2(1.5)$ \\
\hline Helicobacter pylori infection & $82(62.1)$ \\
\hline $\mathrm{LDH}, \mathrm{U} / \mathrm{L}^{*}$ & $160.44 \pm 26.85$ \\
\hline Above normal & $7(5.6)$ \\
\hline$\beta 2$-Microglobulin, mg/L $\mathrm{L}^{\dagger}$ & $1.75 \pm 0.53$ \\
\hline Above normal & $4(4.1)$ \\
\hline EUS evaluation & $70(53.0)$ \\
\hline Mucosa and submucosa & 65 (92.9) \\
\hline Proper muscle & $2(2.9)$ \\
\hline Serosa & $3(4.3)$ \\
\hline \multicolumn{2}{|l|}{ LN involvement } \\
\hline None & $111(84.1)$ \\
\hline Regional LN & $20(15.2)$ \\
\hline Intra-abdominal LN & $1(0.8)$ \\
\hline BM evaluation & $92(69.7)$ \\
\hline Not involved & $88(95.7)$ \\
\hline Involved & $4(4.3)$ \\
\hline \multicolumn{2}{|l|}{ Modified Ann Arbor stage } \\
\hline IE & $108(81.8)$ \\
\hline IE1 & $106(80.3)$ \\
\hline IE2 & $2(1.5)$ \\
\hline IIE & 20 (15.2) \\
\hline IIE1 & 20 (15.2) \\
\hline IIE2 & 0 \\
\hline IV & $4(3.0)$ \\
\hline
\end{tabular}

antrum and angle. Thirty-seven patients (28.0\%) had MALT lymphoma in different parts of stomach at the same time. The most common finding upon endoscopic evaluation was mucosal change, such as erythematous or whitish discoloration, friable
Table 1. Continued

\begin{tabular}{lc}
\hline \multicolumn{1}{c}{ Variable } & Value \\
\hline Modified Ann Arbor stage (disregarding BM involvement) \\
IE & $111(84.1)$ \\
IE1 & $109(82.6)$ \\
IE2 & $2(1.5)$ \\
IIE & $21(16.0)$ \\
IIE1 & $20(15.2)$ \\
IIE2 & $1(0.8)$ \\
Death during follow-up & None \\
\hline
\end{tabular}

Data are presented as median (range), number (\%), or mean \pm SD.

LDH, lactase dehydrogenase; EUS, endoscopic ultrasound; LN, lymph node; $\mathrm{BM}$, bone marrow.

${ }^{*} \mathrm{LDH}$ level was available for $126(95.5 \%)$ of the 132 patients; ${ }^{\dagger} \beta 2$ Microglobin level was available for 98 (74.2\%) of the 132 patients.

mucosa, or focal mucosal irregularity. In rare cases, MALT lymphoma presented as mass (four patients) or polyp (two patients). Evaluation for $H$. pylori infection status was done in all patients with rapid urease test or pathologic confirmation and showed positive result in 82 patients (62.1\%).

LDH and $\beta 2$-microglobulin levels were measured in 126 $(95.5 \%)$ and $98(74.2 \%)$ patients, respectively, and were elevated above normal levels (202 U/L for LDH and $2.4 \mathrm{mg} / \mathrm{L}$ for $\beta 2$ microglobulin) in seven (5.6\%) and four patients (4.1\%). CT scan was performed in all patients, and significant lymph node enlargement was found in 21 patients (16.0\%): 20 (15.2\%) with regional lymph node involvement and one $(0.8 \%)$ with distal intra-abdominal lymph node involvement. EUS was done in 70 patients $(53.0 \%)$, and most of them $(65,92.9 \%)$ had lesions limited to mucosa and submucosa layer (within the third layer on EUS imaging).

\section{Bone marrow involvement}

Among the 132 patients in this study, 92 had bone marrow aspiration and biopsy to rule out bone marrow involvement as one of the initial diagnostic processes. Four patients (4.3\%) were confirmed to have bone marrow involvement. Endoscopic findings of these four patients are shown in Fig. 2, and they all seems superficial lesions. Slides of the bone marrow biopsy samples in three patients that were available in the archive of current institute were reviewed. Scattered or focal lymphocyte aggregates were noted from the immunohistochemistry (IHC) staining for CD 20 (Fig. 3A-C). The extent of bone marrow involvement was less than $10 \%$ of the total evaluated biopsy sample in all patients. PCR test for IgH was performed using bone marrow aspirate in three patients, which did not show definite monoclonality. None of the patients with bone marrow involvement presented with significant systemic symptoms, and two patients complained of nonspecific abdominal symptoms such as epigastric pain or dyspepsia. Other detailed characteristics of these four patients are shown in Table 2. 

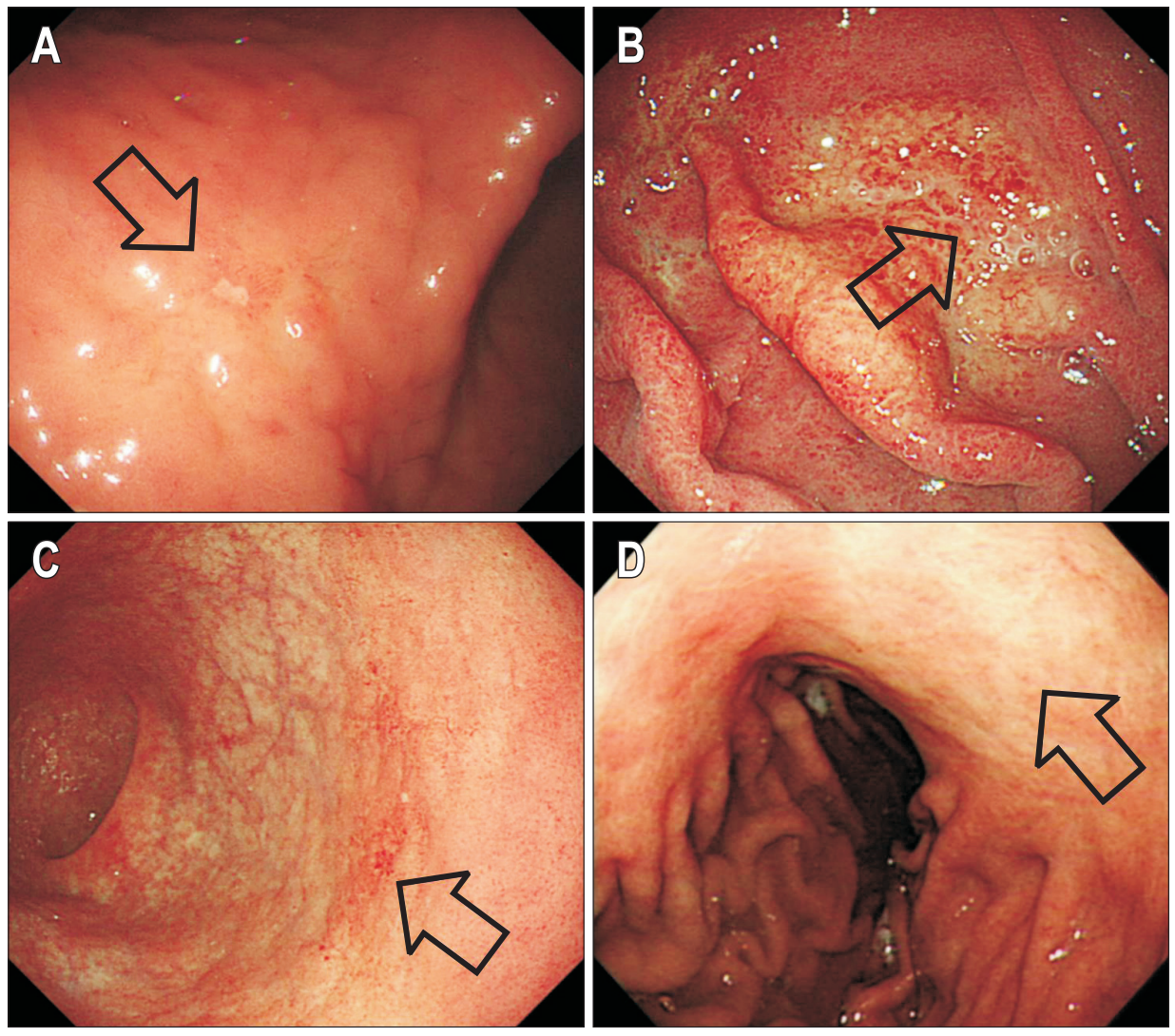

Fig. 2. Endoscopic findings of four patients with bone marrow involvement. Lesions are indicated with black arrow. (A) Nodular elevation with central erosion at the lower body, anterior wall side in patient 1; (B) erythematous flat nodular mucosal change with surrounding whitish discoloration at fundus in patient 2; (C) slightly elevated erythematous change at proximal antrum, greater curvature/posterior wall side in patient 3; and (D) whitish flat discoloration with depression at the mid to lower body, lesser curvature side in patient 4.

\section{Stage}

According to modified Ann Arbor staging system, most of the patients were in stage IE (108, 81.8\%). Among these patients, two patients were confirmed to exhibit invasion beyond the submucosal layer, which is indicative of stage IE2. Twenty patients with regional lymph node involvement were classified as stage IIE1. All four patients in stage IV were classified as such due to bone marrow involvement.

We restaged the patients disregarding the bone marrow involvement in order to determine the differences in baseline characteristics between patients with or without bone marrow involvement. Three patients among the four patients in stage IV did not have any other site of involvement and, thus, were reclassified as stage IE1, while one patient was restaged as IIE2 due to intra-abdominal lymph node involvement beyond regional involvement. (Table 2)

Among the 70 patients who had EUS evaluation, 65 (92.9\%) had MALT lymphoma involvement in mucosa and submucosa layer, putting them into stage T1 according to the Paris staging system, while two patients (2.9\%) were in stage T2, and three patients (4.3\%) were in stage T3.

\section{Treatment and follow-up in patients with bone marrow involvement}

Among the four patients with bone marrow involvement, $H$. pylori infection was confirmed in two patients (patient number 1 and 3) at the time of diagnosis, and H. pylori eradication treatment was administered as initial treatment. Upon the follow-up endoscopic evaluation including biopsy that was done at 3 to 4 months after initial $H$. pylori eradication, patient 1 showed residual MALT lymphoma, while patient 3 exhibited CR with scar change. Because there was no significant symptom or evidence for disease progression or dissemination, patient 1 was followed without immediate treatment for 3 more months. Upon followup evaluation, remission of the residual lesion was confirmed. In patient 3, bone marrow aspiration and biopsy was performed 6 months after $H$. pylori eradication treatment and showed a disappearance of the CD 20-positive lymphocyte aggregates (Fig. 3D).

For patient 2 who did not have $H$. pylori infection and had disease confined to gastric wall if the bone marrow involvement was disregarded, radiation therapy was used as initial treatment (3,060 cGy divided into 17 fractions). However, upon the follow-up endoscopic biopsy which was done 5 months after radiation therapy, residual MALT lymphoma lesion was noted with $H$. pylori infection. $H$. pylori eradication treatment was given using amoxicillin and clarithromycin with pantoprazole as second-line treatment that resulted in CR at 3-month followup evaluation after eradication treatment. Follow-up bone marrow evaluation was not done; however, the patient is being followed twice a year with endoscopy including biopsy without 

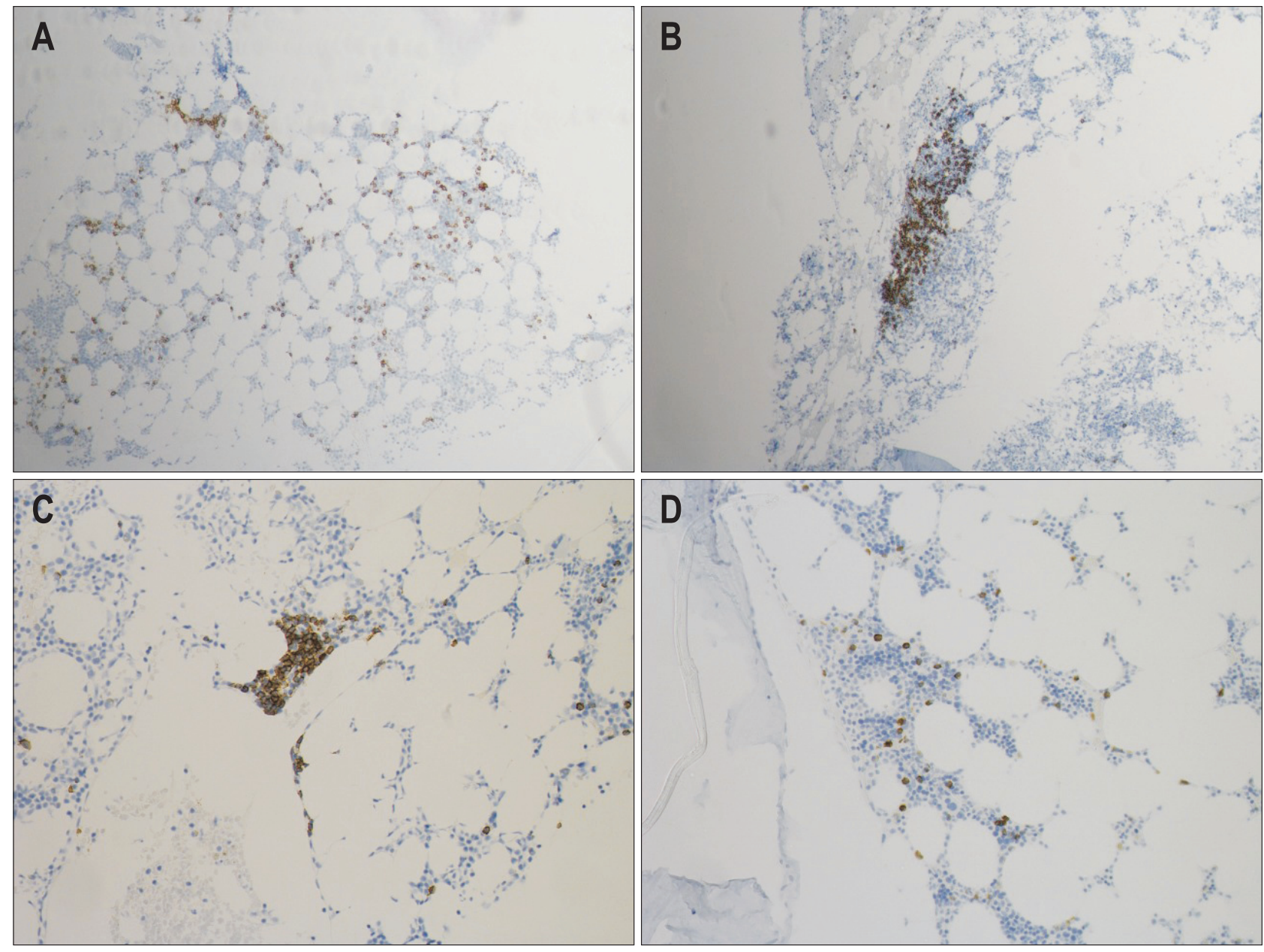

Fig. 3. Immunohistochemistry of $\mathrm{CD} 20$ in bone marrow biopsy samples from three patients. Bone marrow biopsy from (A) left iliac bone of patient 1 (100x, 5\% involvement); (B) right iliac bone of patient 2 (200x, 5\% to 10\% involvement); (C) left iliac bone of patient 3 (200x, 5\% involvement); and (D) left iliac bone of patient 3 at 6 months after the Helicobacter pylori eradication therapy showing no evidence of involvement (200x).

any evidence of disease recurrence as of January 2017.

Patient 4 did not have $H$. pylori infection and had disease stage of IIE2 due to the involvement of regional and para-aortic lymph nodes upon initial CT imaging. Because the patient had abdominal symptoms with multiple lymph node involvement, chemotherapy consisting of cyclophosphamide, doxorubicin, vincristine, and prednisolone (CHOP) was used as the initial treatment. Follow-up CT scan showed persistent lymph node enlargement even after four cycles of initial chemotherapy; and therefore, the regimen was changed to dexamethasone and high-dose cytarabine with cisplatin (DHAP). Endoscopic evaluation that was done 3 months after salvage chemotherapy confirmed remission of MALT lymphoma, and CT scan that was taken 1 year after treatment showed disappearance of the lymph node enlargement. Follow-up bone marrow evaluation was not performed, but the patient is being followed without any evidence of disease recurrence for more than 10 years.

\section{Factors associated with bone marrow involvement}

We tried to performed additional analysis to evaluate the factors associated with bone marrow involvement. However, the number of patients with bone marrow involvement was too small for statistical analysis. The baseline characteristics of the two groups; those with and without bone marrow involvement, are shown in Table 3.

\section{DISCUSSION}

Bone marrow involvement is known to occur in rare cases in patients with MALT lymphoma. Current study showed bone marrow involvement in $4.3 \%$ of the total patients who had undergone bone marrow evaluation at the time of initial diagnosis of MALT lymphoma. In this study, bone marrow involvement was noted even in patients with disease confined to gastric wall without lymph node or other organ involvement.

Primary gastric lymphoma has been reported in approxi- 


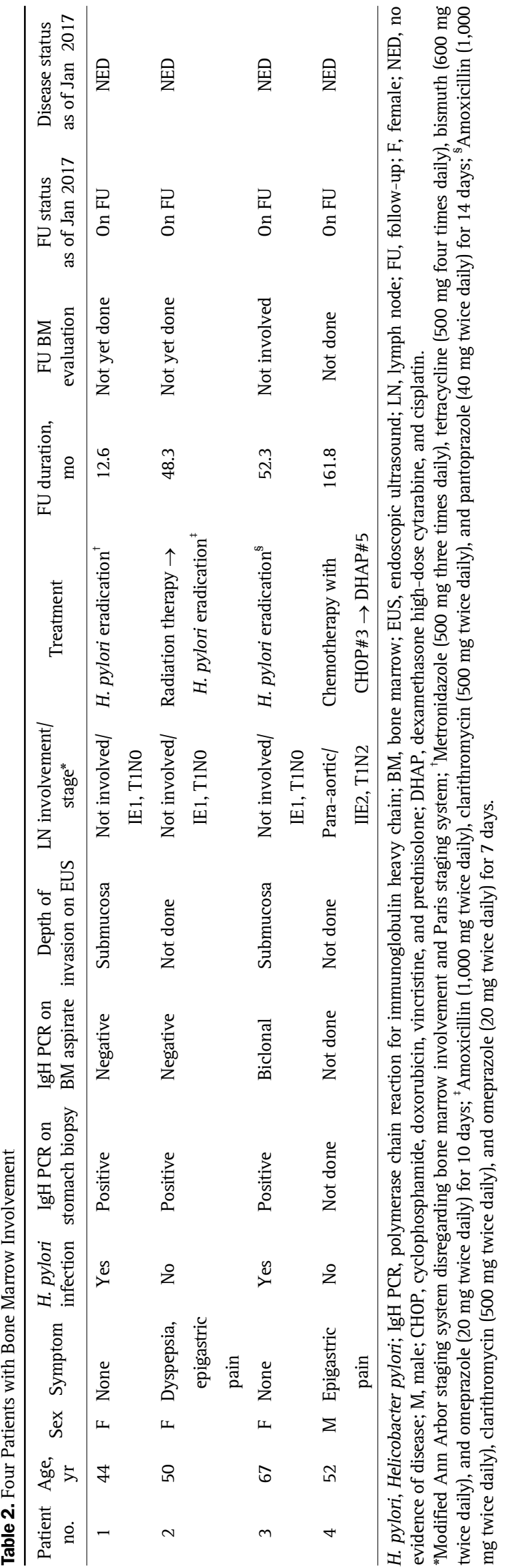

Table 3. Comparison of Baseline Characteristics in Patients with or without Bone Marrow Involvement

\begin{tabular}{lcc}
\hline \multicolumn{1}{c}{ Variable } & $\begin{array}{c}\text { Without BM } \\
\text { involvement }(\mathrm{n}=88)\end{array}$ & $\begin{array}{c}\text { With BM } \\
\text { involvement }(\mathrm{n}=4)\end{array}$ \\
\hline Age, yr & $51.17 \pm 11.0$ & $53.25 \pm 9.8$ \\
LDH, U/L & $158.2 \pm 26.1$ & $148.0 \pm 25.2$ \\
$\beta 2-$ Microglobulin, mg/L & $1.74 \pm 0.59$ & $1.75 \pm 0.21$ \\
Multiplicity on EGD & $44(50)$ & $3(75)$ \\
Helicobacter pylori infection & $56(66.6)$ & $3(75)$ \\
LN involvement & & \\
None & $72(81.8)$ & $3(75)$ \\
Regional LN & $16(18.2)$ & 0 \\
Intra-abdominal LN & 0 & $1(25)$ \\
Depth of invasion on EUS & & \\
T1 & $48 / 52(92.3)$ & $2 / 2(100)$ \\
T2 & $2 / 52(3.8)$ & $0 / 2(0)$ \\
T3 & $2 / 52(3.8)$ & $0 / 2(0)$ \\
\hline
\end{tabular}

Data are presented as mean $\pm \mathrm{SD}$, number $(\%)$, or number/number $(\%)$. $\mathrm{BM}$, bone marrow; LDH, lactase dehydrogenase; EGD, esophagogastroduodenoscopy; LN, lymph node; EUS, endoscopic ultrasound. ${ }^{*} \mathrm{LDH}$ was available for 86 (without BM involvement) and 3 patients (with BM involvement); ${ }^{\dagger} \beta 2$-Microglobulin was available for 65 (without BM involvement) and 2 patients (with BM involvement).

mately $0.05 \%$ of patients who go through routine screening endoscopy for gastric cancer in South Korea, ${ }^{25}$ and MALT lymphoma comprises approximately 56\% of the total cases. ${ }^{26}$ In recent retrospective studies conducted in two hospitals in South Korea, bone marrow involvement was reported in $0.5 \%$ to $1.0 \%$ of MALT lymphoma patients, ${ }^{13,14}$ which is lower than what we observed in this study.

Several study groups have suggested similar but different guidelines for diagnosis and treatment of gastric MALT lymphoma, especially in regards to the need for bone marrow biopsy with or without aspiration and $H$. pylori eradication therapy. The National Comprehensive Cancer Network suggests that bone marrow study may be useful in selected cases, presumably in patients who are suspected to have advanced stage disease. They recommend $H$. pylori eradication treatment in cases of early stage disease (i.e., stage IE and IIE) with proven $H$. pylori infection. However, systemic induction chemo-immunotherapy or locoregional radiation therapy has been suggested in specific settings in patients who satisfy the treatment indication regardless of $H$. pylori infection status: presence of symptoms, gastrointestinal bleeding, threatened end-organ function, bulky disease, steady progression of disease, and patient's preference. ${ }^{15}$ European Society for Medical Oncology (ESM0) recommends bone marrow aspiration and biopsy in all patients who present with MALT lymphoma. According to this guideline, $H$. pylori eradication treatment must be given as initial treatment to all gastric MALT lymphoma patients, independent of initial stage and $H$. pylori infection status since occasional response has 
been reported even in cases without proven $H$. pylori infection. Additional treatment using chemotherapeutic agents is suggested in symptomatic advanced stage disease with certain indications: overt progression, bulky disease, impending organ damage or patient's preference. ${ }^{16}$ However, European GastroIntestinal Lymphoma Study (EGILS) group does not recommend bone marrow biopsy as an initial diagnostic procedure but suggests $H$. pylori eradication therapy as initial treatment in all gastric MALT lymphoma patients independent of the stage. They suggest performing bone marrow evaluation only in the case of failure of lymphoma regression after $H$. pylori eradication and before initiating oncologic treatment. ${ }^{17}$

The results of current study imply that systemic chemotherapy may be deferred in patients with gastric MALT lymphoma confined to gastric wall even with bone marrow involvement and the presence of nonspecific symptoms which could be possibly associated with disease (i.e., epigastric pain or dyspepsia) if they do not have any signs of symptoms related to bone marrow involvement, considering its indolent disease behavior and excellent treatment outcome with loco-regional treatment including $H$. pylori eradication therapy. This finding concurs with the guidelines suggested by the ESMO and EGILS groups. Furthermore, because bone marrow involvement did not significantly change the initial treatment option, we may assume that bone marrow evaluation may not need to be included in the initial diagnostic process.

Upon review of our bone marrow biopsy slides from patients with MALT lymphoma, we were able to find out that the extent of marrow involvement was at most 10\% of the total examined area. This triviality of involvement is also supported by PCR result, which did not show definite monoclonality on bone marrow aspiration while clonality was confirmed on biopsy samples from primary site. This may partly explain the absence of significant systemic symptoms in patients who were confirmed to have bone marrow involvement. However, this may raise questions regarding the sensitivity of bone marrow biopsy in evaluating bone marrow involvement due to the fact that we can examine only a small portion of bone marrow through conventional bone marrow aspiration and biopsy technique. There is still possibility of undetected portion of patients with marrow involvement, but a true sensitivity and false-negative rate for bone marrow aspiration and biopsy have not been reported yet because there is no gold standard test to evaluate true marrow involvement. Nevertheless, we must assume that the previously and currently reported figures on the proportion of patients with bone marrow involvement might have been underestimated due to subtle marrow involvement. Meanwhile, a previous study suggested that determination of the presence of bone marrow (BM) involvement could constitute overdiagnosis and that subtle CD20 positivity in the BM should not be regarded as evidence of BM involvement. ${ }^{20}$ Our data support this suggestion since the extent of BM involvement was 5\% to 10\% in all cases with BM involvement. This suggest that cases of MALT lymphoma with BM invasion may actually have subtle CD20 positivity in the BM that may not change clinical course of the patients. Consensus is needed in terms of diagnostic criteria of BM involvement in the future.

Risk factors for bone marrow involvement have not been clearly identified so far. Some studies have shown that the patients with $H$. pylori negative MALT lymphoma had more advanced disease compared to $H$. pylori positive MALT lyhmphoma, ${ }^{27-29}$ while another study suggested possible relation between the presence of monoclonal gammopathy and CD5 positivity with bone marrow involvement. ${ }^{30}$ We can assume that the rarity of MALT lymphoma itself and associated bone marrow involvement has eventually resulted in small sample size, making it difficult to delineate any potential risk factor for marrow involvement. Further study with larger number of patients is warranted to deal with this specific issue, including the possible association between the bone marrow involvement and lymph node involvement.

There are some limitations in this study. Because this was a retrospective study, there are some missing data in the initial diagnostic evaluation, such as LDH and $\beta 2$-microglobulin levels. Furthermore, EUS and bone marrow aspiration and biopsy were not performed in all patients. This may limit the statistical significance of current analyses; however, we are reporting more patients with bone marrow involvement than other recently published studies performed on patients with gastric MALT lymphoma. Also, due to the retrospective nature of current study, we were not able to give $H$. pylori eradication therapy in a protocol-based manner. Two patients with bone marrow involvement received amoxicillin and clarithromycin with proton pump inhibitor, while one patient was treated with quadruple treatment comprising metronidazole, tetracycline, bismuth and proton pump inhibitor. However, we confirmed successful $H$. pylori eradication through follow-up biopsy and rapid urease test that was performed at least 6 weeks after eradication therapy in all three patients. There are still no concrete reports that the choice of regimen for $H$. pylori eradication plays a significant role in the disease response and outcome of gastric MALT lymphoma. In current study, the proportion of patients with $H$. pylori infection was about $60 \%$, which is lower than the figures reported in other studies. This may be due to frequent empirical prescription of proton pump inhibitor to patients with abdominal symptom in local clinics, possible previous treatment for $H$. pylori infection, or not incorporating other modalities such as $H$. pylori culture or PCR method to identify $H$. pylori infection status. Data to better elaborate this matter is lacking due to retrospective nature of this study. Finally, current study report higher proportion of patient with regional lymph node involvement compared to recently published data. ${ }^{13,14}$ However, considering some other data, ${ }^{31,32}$ this may partly due to different criteria that was used to diagnose lymph node involvement in 
different studies. Further study is warranted to standardize the staging process of gastric MALT lymphoma including diagnostic criteria of lymph node involvement.

In summary, we were able to find out that approximately $4.3 \%$ of patients with gastric MALT lymphoma had bone marrow involvement, but their involvement was trivial, with at most $10 \%$ of the total evaluated biopsy sample area. Additionally, we were able to show that the patients with disease confined to gastric wall were less likely to have bone marrow involvement compared to those with more disseminated disease. And locoregional treatments such as $H$. pylori eradication and radiation therapy were sufficient to induce CR of MALT lymphoma even in patients with bone marrow involvement if the patient had disease confined to gastric wall.

Based on these findings, we may consider administering loco-regional treatment before systemic chemotherapy even in patients with bone marrow involvement if a patient has disease limited to stomach with solitary bone marrow involvement. In addition, we can also propose that bone marrow examination may be deferred due to the fact that marrow involvement may not change the treatment options and outcome of the patients with gastric MALT lymphoma confined to stomach wall without systemic symptoms.

\section{CONFLICTS OF INTEREST}

No potential conflict of interest relevant to this article was reported.

\section{ACKNOWLEDGEMENTS}

This study was supported by a grant from the National Cancer Center, Korea (\# 1610160-2) and the National Research Foundation, Korea (\#NRF-2016R1A2B1010377).

\section{REFERENCES}

1. Harris NL, Jaffe ES, Stein H, et al. A revised European-American classification of lymphoid neoplasms: a proposal from the International Lymphoma Study Group. Blood 1994;84:1361-1392.

2. Swerdlow SH, Campo E, Pileri SA, et al. The 2016 revision of the World Health Organization classification of lymphoid neoplasms. Blood 2016;127:2375-2390.

3. A clinical evaluation of the International Lymphoma Study Group classification of non-Hodgkin's lymphoma: the non-Hodgkin's lymphoma classification project. Blood 1997;89:3909-3918.

4. Wotherspoon AC, Ortiz-Hidalgo C, Falzon MR, Isaacson PG. Helicobacter pylori-associated gastritis and primary B-cell gastric lymphoma. Lancet 1991;338:1175-1176.

5. Hyjek E, Smith WJ, Isaacson PG. Primary B-cell lymphoma of salivary glands and its relationship to myoepithelial sialadenitis. Hum Pathol 1988;19:766-776.
6. Hyjek E, Isaacson PG. Primary B cell lymphoma of the thyroid and its relationship to Hashimoto's thyroiditis. Hum Pathol 1988;19:1315-1326.

7. Thieblemont C, Bastion Y, Berger F, et al. Mucosa-associated lymphoid tissue gastrointestinal and nongastrointestinal lymphoma behavior: analysis of 108 patients. J Clin Oncol 1997;15:16241630.

8. Zucca E, Bertoni F, Vannata B, Cavalli F. Emerging role of infectious etiologies in the pathogenesis of marginal zone B-cell lymphomas. Clin Cancer Res 2014;20:5207-5216.

9. Isaacson PG. The MALT lymphoma concept updated. Ann Oncol 1995;6:319-320.

10. Zucca E, Bertoni F, Roggero E, Cavalli F. The gastric marginal zone B-cell lymphoma of MALT type. Blood 2000;96:410-419.

11. Zucca E, Stathis A, Bertoni F. The management of nongastric MALT lymphomas. Oncology (Williston Park) 2014;28:86-93.

12. Lee MH, Jung HY, Kang GH, et al. Two cases of gastric low-grade mucosa-associated lymphoid tissue lymphoma involving bone marrow. Korean J Gastroenterol 2000;36:695-700.

13. Min BH, Park JY, Kim ER, et al. Limited role of bone marrow aspiration and biopsy in the initial staging work-up of gastric mucosa-associated lymphoid tissue lymphoma in Korea. Gut Liver 2014;8:637-642.

14. Park JY, Kim SG, Kim JS, Jung HC. Bone marrow involvement is rare in superficial gastric mucosa-associated lymphoid tissue lymphoma. Dig Liver Dis 2016;48:81-86.

15. Zelenetz AD, Gordon LI, Wierda WG, et al. B-cell lymphoma version 1. 2017, NCCN guidelines [Internet]. Fort Washington: National Comprehensive Cancer Network [cited 2017 Jan 16]. Available from: https://www.nccn.org/professionals/physician_gls/pdf/ b-cell.pdf.

16. Zucca E, Copie-Bergman C, Ricardi U, et al. Gastric marginal zone lymphoma of MALT type: ESMO clinical practice guidelines for diagnosis, treatment and follow-up. Ann Oncol 2013;24 Suppl 6:vi144-vi148.

17. Ruskoné-Fourmestraux A, Fischbach W, Aleman BM, et al. EGILS consensus report: gastric extranodal marginal zone B-cell lymphoma of MALT. Gut 2011;60:747-758.

18. Wotherspoon AC, Doglioni C, Diss TC, et al. Regression of primary low-grade B-cell gastric lymphoma of mucosa-associated lymphoid tissue type after eradication of Helicobacter pylori. Lancet 1993;342:575-577.

19. Konda B, Pathak S, Edwin I, et al. Safe and successful bone marrow biopsy: an anatomical and CT-based cadaver study. Am J Hematol 2014;89:943-946.

20. Won D, Park CJ, Shim H, et al. Subtle CD20 positivity in the bone marrow of a patient who has a mucosa-associated lymphoid tissue lymphoma should not be regarded as evidence of involvement in the bone marrow. Histopathology 2013;62:397-405.

21. Radaszkiewicz T, Dragosics B, Bauer P. Gastrointestinal malignant lymphomas of the mucosa-associated lymphoid tissue: factors relevant to prognosis. Gastroenterology 1992;102:1628-1638. 
22. Ruskoné-Fourmestraux A, Dragosics B, Morgner A, Wotherspoon A, De Jong D. Paris staging system for primary gastrointestinal lymphomas. Gut 2003;52:912-913.

23. Choi D, Lim HK, Lee SJ, et al. Gastric mucosa-associated lymphoid tissue lymphoma: helical CT findings and pathologic correlation. AJR Am J Roentgenol 2002;178:1117-1122.

24. Copie-Bergman C, Gaulard P, Lavergne-Slove A, et al. Proposal for a new histological grading system for post-treatment evaluation of gastric MALT lymphoma. Gut 2003;52:1656.

25. Yang HJ, Lee C, Lim SH, et al. Clinical characteristics of primary gastric lymphoma detected during screening for gastric cancer in Korea. J Gastroenterol Hepatol 2016;31:1572-1583.

26. Kim JM, Ko YH, Lee SS, et al. WHO classification of malignant lymphomas in Korea: report of the third nationwide study. Korean J Pathol 2011;45:254-260.

27. Chung SJ, Kim JS, Kim H, et al. Long-term clinical outcome of helicobacter pylori-negative gastric mucosa-associated lymphoid tissue lymphoma is comparable to that of H. pylori-positive lymphoma. J Clin Gastroenterol 2009;43:312-317.

28. Nakamura S, Matsumoto T, Ye H, et al. Helicobacter pylori- negative gastric mucosa-associated lymphoid tissue lymphoma: a clinicopathologic and molecular study with reference to antibiotic treatment. Cancer 2006;107:2770-2778.

29. Gong EJ, Ahn JY, Jung HY, et al. Bone marrow involvement is not associated with the clinical outcomes of gastric mucosa-associated lymphoid tissue lymphoma. Scand J Gastroenterol 2016;51:942948.

30. Conlan MG, Bast M, Armitage J0, Weisenburger DD. Bone marrow involvement by non-Hodgkin's lymphoma: the clinical significance of morphologic discordance between the lymph node and bone marrow. Nebraska Lymphoma Study Group. J Clin Oncol 1990;8:1163-1172.

31. Thieblemont C. Clinical presentation and management of marginal zone lymphomas. Hematology Am Soc Hematol Educ Program 2005:2005;307-313.

32. Montalbán C, Castrillo JM, Abraira V, et al. Gastric B-cell mucosaassociated lymphoid tissue (MALT) lymphoma: clinicopathological study and evaluation of the prognostic factors in 143 patients. Ann Oncol 1995;6:355-362. 